\title{
Clinical considerations in transitioning patients with epilepsy from clonazepam to clobazam: a case series
}

\author{
Raman Sankar ${ }^{1 *}$, Steve Chung ${ }^{2}$, Michael Scott Perry ${ }^{3}$, Ruben Kuzniecky ${ }^{4}$ and Saurabh Sinha ${ }^{5}$
}

\begin{abstract}
Introduction: In treating refractory epilepsy, many clinicians are interested in methods used to transition patients receiving clonazepam to clobazam to maintain or increase seizure control, improve tolerability of patients' overall drug therapy regimens, and to enhance quality of life for patients and their families. However, no published guidelines assist clinicians in successfully accomplishing this change safely.

Case presentations: The following three case reports provide insight into the transition from clonazepam to clobazam. First, an 8-year-old Caucasian boy with cryptogenic Lennox-Gastaut syndrome beginning at 3.5 years of age, who was experiencing multiple daily generalized tonic-clonic, absence, myoclonic, and tonic seizures at presentation. Second, a 25-year-old, left-handed, White Hispanic man with moderate mental retardation and medically refractory seizures that he began experiencing at 1 year of age, secondary to tuberous sclerosis. When first presented to an epilepsy center, he had been receiving levetiracetam, valproate, and clonazepam, but reported having ongoing and frequent seizures. Third, a 69-year-old Korean woman who had been healthy until she had a stroke in 2009 with subsequent right hemiparesis; as a result, she became less physically and socially active, and had her first convulsive seizure approximately 4 months after the stroke.

Conclusions: From these cases, we observe that a rough estimate of final clobazam dosage for each mg of clonazepam under substitution is likely to be at least 10-fold, probably closer to 15-fold for many patients, and as high as 20-fold for a few. Consideration and discussion of the pharmacokinetic, pharmacologic, and clinical properties of 1,4- and 1,5-benzodiazepine action provide a rationale on why and how these transitions were successful.
\end{abstract}

Keywords: Clobazam, Clonazepam, Switching, Transition

\section{Introduction}

Benzodiazepines have been used to treat seizures since 1965, when a group from Marseilles, led by Henri Gastaut, reported on the successful use of diazepam to treat nonconvulsive (mainly absence) seizures [1] and its utility in the treatment of status epilepticus [2]. Prior to that, benzodiazepines such as diazepam and chlordiazepoxide had been employed primarily as anxiolytics and hypnotics. Despite the observations by Gastaut, the use of diazepam for absence epilepsy did not gain traction because the

\footnotetext{
* Correspondence: RSankar@ucla.edu

'Division of Pediatric Neurology, David Geffen School of Medicine at UCLA, University of California-Los Angeles, Room 22-474 MDCC, Los Angeles, CA 90095-1752, USA

Full list of author information is available at the end of the article
}

1960s marked the emergence of succinimides such as ethosuximide, which seemed to be less sedating and better tolerated than diazepam. Subsequently, Henri Gastaut published that clonazepam was even more effective than diazepam in the treatment of status epilepticus [3].

The initial approval of clonazepam by the US Food and Drug Administration (FDA), as reviewed by Thomas Brown [4], suggested that it be used for the following types of seizures: absence (typical petit mal), infantile spasms (infantile myoclonic, massive spasms, salaam), atypical absence (atypical petit mal), akinetic (astatic, atonic, drop attack), "minor motor" attacks, and "Lennox-Gastaut syndrome," (LGS) but not for the treatment of grand mal, partial seizures with complex symptoms (for example, psychomotor, temporal lobe), or 
focal seizures. The more recent version of the prescribing information is more restrictive and states that, "It is useful alone or as an adjunct in the treatment of the LGS (petit mal variant), akinetic and myoclonic seizures. In patients with absence seizures (petit mal) who have failed to respond to succinimides, clonazepam (Klonopin ${ }^{\mathrm{Tw}}$ ) may be useful [5]." Of interest, the initial experience described in the European literature was based on the use of intravenous clonazepam, which is not available in the USA.

Until the approval of clobazam $\left(\mathrm{Onfi}^{\mathrm{m}}\right)$ in the USA in October 2011 for adjunctive treatment of seizures associated with LGS [6], benzodiazepines had been used mainly as rescue medications for status epilepticus or acute repetitive seizures. In addition to concerns about excessive sedation, long-term benzodiazepine use raises questions about maintenance of efficacy because of potential development of tolerance. The indications section of the FDA prescribing information for clonazepam indicates, "In some studies, up to $30 \%$ of patients have shown a loss of anticonvulsant activity, often within 3 months of administration. In some cases, dosage adjustment may reestablish efficacy [5]."

In addition, of the various classes of anticonvulsant medications, benzodiazepines have been especially implicated in producing cognitive and behavioral adverse effects $[7,8]$. However, clobazam, a 1,5-benzodiazepine, seems to differ in its spectrum of pharmacologic activities from traditional 1,4-benzodiazepines [9,10]. Indeed, a recent study provided sustained efficacy results for clobazam in patients with LGS over 3 years with stable dosing [11].

Clinicians are considerably interested in switching some patients receiving clonazepam to clobazam to maintain or improve seizure control, while also improving tolerability and quality of life for the patient. However, no published guidelines assist clinicians in successfully accomplishing this change safely. In this report, we provide three case reports on this therapy transition, and summarize in the following discussion the known pharmacokinetic, pharmacologic, and clinical aspects of benzodiazepine action to arrive at a successful therapeutic solution to this problem.

\section{Case presentations}

\section{Case 1 (8-year-old Caucasian boy with cryptogenic LGS)}

Case 1 is an 8-year-old Caucasian boy with cryptogenic LGS beginning at 3.5 years of age. An electroencephalograph (EEG) showed diffuse slowing, with a generalized slow spike-and-wave pattern typical of LGS. His magnetic resonance imaging (MRI) was normal. At the time of presentation, he experienced multiple daily generalized tonic-clonic (GTC), absence, myoclonic, and tonic seizures. He had failed several prior antiepileptic drugs including levetiracetam (maximum dosage unknown), lamotrigine $(7 \mathrm{mg} / \mathrm{kg} /$ day $)$, the ketogenic diet, and vagal nerve stimulation (VNS, output current $2.5 \mathrm{~mA}$, on time:
21 seconds, off time: 0.8 minutes). A physical examination revealed a thin boy, unable to walk or speak with many healing abrasions from frequent seizure-induced falls. At the time clobazam was added to his treatment regimen, he was failing zonisamide $(10 \mathrm{mg} / \mathrm{kg} /$ day), valproate $(42 \mathrm{mg} / \mathrm{kg} /$ day, concentration $103 \mu \mathrm{g} / \mathrm{mL})$, rufinamide $(50 \mathrm{mg} / \mathrm{kg} /$ day), and clonazepam $1.5 \mathrm{mg}$ twice daily.

Clobazam was initiated at $5 \mathrm{mg}$ daily $(0.25 \mathrm{mg} / \mathrm{kg} /$ day $)$ and increased weekly by $5 \mathrm{mg}$ until he reached $10 \mathrm{mg}$ twice daily $(1 \mathrm{mg} / \mathrm{kg} /$ day $)$ over 4 weeks. At this dosing, he became free of tonic and myoclonic seizures, with more than a $90 \%$ reduction of GTC and absence seizures. However, his parents reported significant somnolence, with only 6 hours of wake time each day. Clonazepam weaning was initiated by decreasing dosage by $0.5 \mathrm{mg}$ each week, until clonazepam was entirely discontinued by the end of 6 weeks. As clonazepam was weaned, somnolence resolved, and he continued the same good seizure control.

Rufinamide was subsequently discontinued without incident. Clobazam was titrated to $20 \mathrm{mg}$ twice daily $(2 \mathrm{mg} /$ $\mathrm{kg} /$ day) 2 years after initiation and secondary to recurrent GTC seizures, which occurred in the setting of VNS dysfunction. Now, 3 years after the initiation of clobazam, he experiences rare myoclonic seizures and is able to walk independently, attend school, and communicate with his family.

Case 2 (25-year-old, left-handed, White Hispanic man with moderate mental retardation and medically refractory seizures)

Case 2 is a 25-year-old, left-handed, White Hispanic man with moderate mental retardation and medically refractory seizures that he began experiencing at age 1 year, secondary to tuberous sclerosis. His seizures commence typically with sudden body stiffening for 30 seconds with ictal cry, followed by tonic-clonic seizure, on occasion. Seizure frequency had increased over time, and he often experienced seizures three to four times per day. He had been treated previously with carbamazepine, phenytoin, phenobarbital, lamotrigine, and topiramate. Approximately 5 years ago, he underwent vagus nerve stimulator implantation, which improved his seizures only slightly.

When he first presented to an epilepsy center, he had been receiving levetiracetam, valproate, and clonazepam, but reported having ongoing and frequent seizures. His neurologic examination showed moderate cognitive difficulty, slurred speech, and mild right-arm weakness. Further, his right hand was smaller than his left. He was able to ambulate on his own, but swayed left and right with wide-base stance. His previous EEG showed diffuse background slowing, which was focally more in the left hemisphere. Interictal epileptiform discharges were detected mainly from the left frontotemporal region, along 
with occasional sharp discharges from the right hemisphere. His brain MRI showed subependymal nodules bilaterally and transmantle dysplasia over the left parietal lobe. Initial scalp ictal EEG indicated diffuse bilateral seizure onset during his initial body stiffening. However, better ictal progression was observed over the left hemisphere just prior to the progression of tonic-clonic phase. A subsequent intracranial grid electrode guided study revealed diffuse seizure onset, but maximally over the left frontal and parietal lobes near the transmantle dysplasia.

He underwent surgical resection of the dysplasia, but his seizures did not improve significantly. Because of his frequent seizures, clobazam was started and gradually replaced clonazepam, as indicated in Table 1.

His family reported that his seizure improvement was notable at Week 2, and his seizures became milder and less frequent ( $>80 \%$ reduction), without significant adverse effects from clobazam. During the next 6 months, his clobazam dosage was gradually increased to $15 \mathrm{mg}$ twice daily. His seizure frequency further improved to one to two per week. Moreover, 1 month after his clobazam dosage increase, his levetiracetam dosage was decreased by $50 \%$. He and his family are quite happy with the improvement, and had reported that he has become more active.

\section{Case 3 (69-year-old Korean woman with convulsive seizures)}

Case 3 is a 69-year-old Korean woman who had been quite healthy until she had a stroke in 2009, with subsequent right hemiparesis. Following her stroke, she became less active physically and socially, and approximately 4 months after the stroke, she had her first convulsive seizure. Since her first seizure, she has also noted balance difficulty and worsening of right-leg weakness, requiring use of a walker and a wheelchair. She was placed on phenytoin, and later on oxcarbazepine. Because of imbalance, oxcarbazepine was changed to levetiracetam. On her subsequent visit, her friend reported significant mood changes and anger outbursts, and

Table 1 Dosing transition from clonazepam to clobazam
in a 25-year-old, left-handed White Hispanic man with
moderate mental retardation and medically refractory
seizures
$\begin{array}{ll}\text { Week } 0 & \text { Clonazepam } 1 \mathrm{mg} \mathrm{BID} \\ \text { Week } 1 & \text { Clonazepam } 0.5 \mathrm{mg} \mathrm{BID;} \text { Clobazam } 5 \mathrm{mg} \text { qHS } \\ \text { Week } 2 & \text { Clonazepam } 0.5 \mathrm{mg} \text { qHS; Clobazam } 5 \mathrm{mg} \mathrm{BID} \\ \text { Week } 3 & \text { Clonazepam discontinued; Clobazam } 5 \mathrm{mg} \text { qAM } \\ \end{array}$

BID, twice daily; qAM, every morning; qHS, at bedtime; qPM, every night. she was changed from levetiracetam to lamotrigine gradually. She did not have recurrent seizures with lamotrigine.

However, because of worsening of dizziness at $100 \mathrm{mg}$ daily, lamotrigine was changed to valproate. Her balance issues improved, but she developed hand tremors and recurrent seizures, which often started with olfactory auras, anxiety, and confusion, typically lasting 1 to 2 minutes. On her following clinic visit, she reported another convulsive seizure, which resulted in falling to the ground. In addition, she continued to experience two to three seizures per week. Her MRI scan showed large encephalomalacia over the left frontoparietal region, and an EEG showed left-sided interictal epileptiform discharges, superimposed on background slowing over the left hemisphere. Primarily to address her anxiety, which at times could be associated with her seizures, she was started on clonazepam ( $1 \mathrm{mg} /$ day). Although her anxiety and seizures had improved, she was not able to tolerate clonazepam, often experiencing significant daytime drowsiness. Next, her clonazepam therapy was gradually replaced by clobazam over 2 weeks as described in Table 2 .

With clobazam $10 \mathrm{mg} /$ day, she felt much better cognitively and reported seizure freedom for 3 months initially. Subsequently, she reported approximately one aura every 2 months, and she decided to continue her clobazam dosage without further increase. She did not have significant adverse effects, but felt transient somnolence for the first 2 weeks of her medication transition. She has reported doing much better with balance and seizure control.

\section{Discussion}

The three cases represent distinct patterns of a clonazepam to clobazam transition. In the first case, the addition and titration of the clobazam dosage preceded the weaning of clonazepam. In the second and third cases, clonazepam was safely weaned as clobazam was added and titrated simultaneously. Each case highlights both improved seizure control as well as decreased adverse effects following the change from clonazepam to clobazam.

A study published in 1982 compared the preclinical efficacy and the protective index (PI = toxic dose in 50\% of the population/effective dose in $50 \%$ of the population) of clobazam to those of diazepam, valproate, and

Table $\mathbf{2}$ Dosing transition from clonazepam to clobazam
in a $\mathbf{6 9 - y e a r - o l d ~ K o r e a n ~ w o m a n ~ w h o ~ h a d ~ e x p e r i e n c e d ~ a ~}$
stroke in $\mathbf{2 0 0 9}$ and subsequent right hemiparesis leading
to convulsive seizures
$\begin{array}{ll}\text { Week } 0 & \text { Clonazepam } 1 \mathrm{mg} \mathrm{qHS} \\ \text { Week } 1 & \text { Clonazepam } 0.5 \mathrm{mg} \mathrm{qHS} \text {; Clobazam } 5 \mathrm{mg} \mathrm{qHS} \\ \text { Week } 3 & \text { Clonazepam discontinued; Clobazam 10mg qHS }\end{array}$

$\mathrm{qHS}$, at bedtime. 
phenobarbital and concluded that clobazam performed superiorly in rodent models of induced seizures [12]. Similar advantages for clobazam over 10 different 1,4-benzodiazepines were documented in a study of both anxiolytic performance and antiseizure effect, as measured by electroshock convulsions in rodents compared with sedation and myorelaxant properties [13]. However, this advantage in PI was not discernible in the kindling model [14].

The advantages of clobazam over diazepam in human adaptive performance and reaction time have been studied $[15,16]$. Nicholson suggested, based on these studies, that diazepam and its hydroxylated metabolites (temazepam, oxazepam, and nordiazepam) were suitable for use as hypnotics, while clobazam could be a better daytime anxiolytic [16]. In fact, the binding of clobazam and its metabolite, $N$-desmethylclobazam, to $\gamma$-aminobutyric acid (GABA) receptors in in vitro expression systems demonstrates that they have significantly greater binding affinities for $\alpha_{2}$ - versus $\alpha_{1}$-subunit-containing GABA receptor complexes, a difference not observed for clonazepam, for which no distinction between $\alpha_{2}$ and $\alpha_{1}$ receptors was observed (Figure 1) [10]. Many lines of evidence suggest that GABA receptors containing $\alpha_{1}$ subunits mediate sedation (as is known for zolpidem), while substantial anticonvulsant activity can be achieved by activation of GABA receptors containing $\alpha_{2}$ subunits [9].
For development of tolerance, there appears to be differences among species and models compared with humans with epilepsy. Thus, in amygdala-kindled rats, tolerance to clobazam developed more rapidly than tolerance to clonazepam [17]. In acute seizures induced by pentylenetetrazole or maximal electroshock, rats chronically treated with diazepam, clonazepam, or clobazam for 28 days and tested over the ensuing 2 weeks showed similar extents of tolerance [18].

In a prospective Canadian study of 115 children with varying types of epilepsy treated with clobazam, the 79 responders developed at least some degree of tolerance over a 24-month period [19]. The same study also reported that 25 of 37 (68\%) children who had failed to respond to 1,4-benzodiazepines (that is, nitrazepam or clonazepam) had responded to the 1,5-benzodiazepine clobazam. In addition, the use of clobazam appeared to be associated with less neurotoxicity than was observed for the 1,4-benzodiazepines clonazepam and nitrazepam. Another Canadian study, involving adult patients with different types of epilepsy, observed that those who had achieved a sustained response to clobazam differed from those who had developed tolerance in duration of epilepsy and the clobazam concentrations that had been maintained [20]. Sustained responders had experienced a shorter duration of epilepsy (mean 16.5 versus 24.5
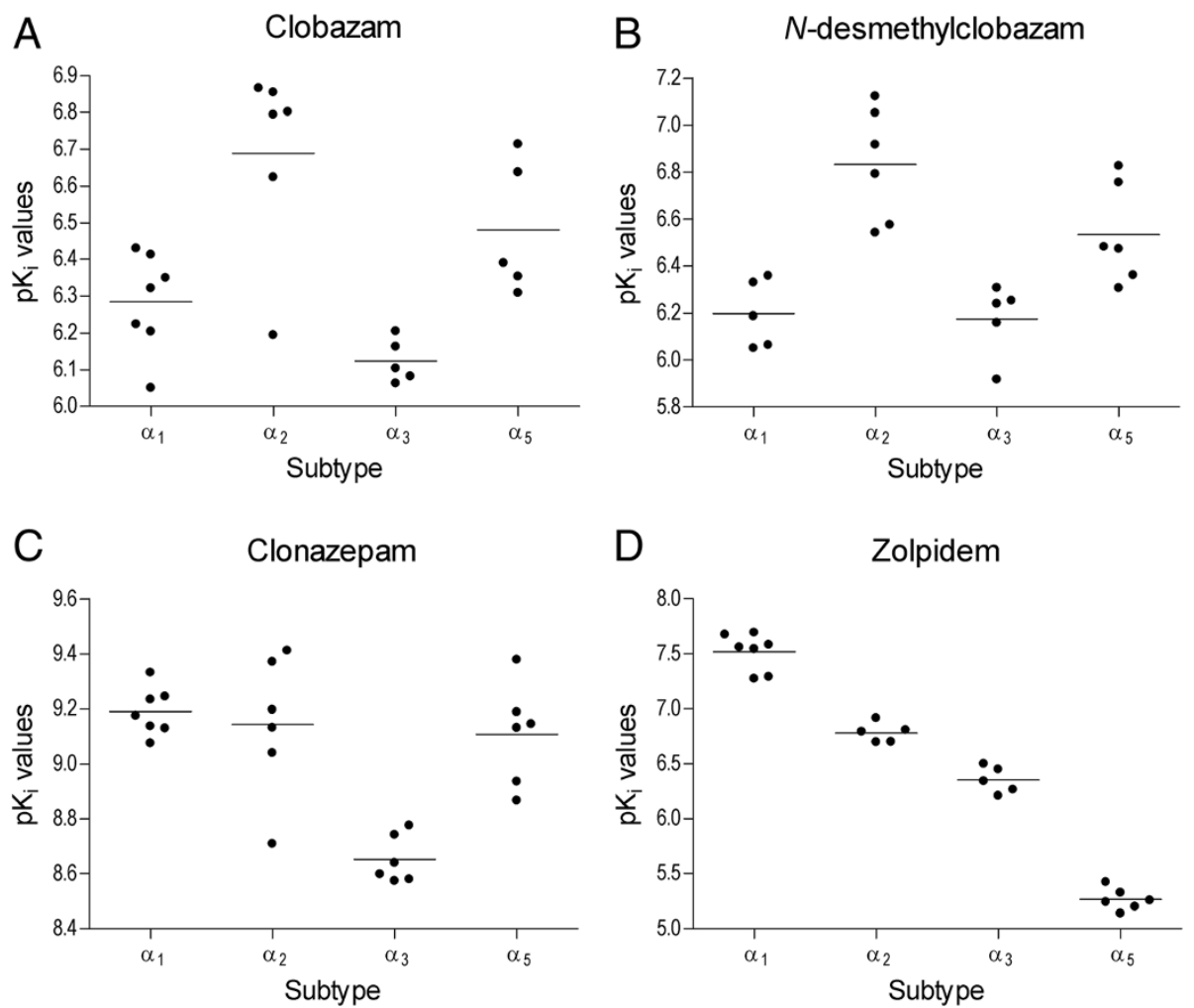

Figure 1 Distribution of individually determined $\mathrm{pK}_{\mathrm{i}}$ values for (A) clobazam, (B) N-desmethylclobazam, (C) clonazepam, and (D) zolpidem across GABAA-receptor subtypes. From Jensen et al. [10]. $K_{i}$ inhibition constant. 
years, $\mathrm{p}=0.015)$ and had attained greater clobazam concentrations ( 0.50 versus $0.22 \mu \mathrm{M}, \mathrm{p}=0.017$ ).

A human study followed patients who participated in Phase II and III LGS studies into an extended open-label phase. Of 74 patients who had achieved at least a $50 \%$ reduction in drop seizures from baseline to Month 3, 64 (86\%) maintained this degree of response with stable dosages over time for up to 3 years [11]. In a subset of patients in that study for whom data were available for up to 5 years, the mean modal clobazam dosage was $0.97 \mathrm{mg} / \mathrm{kg} /$ day compared with $0.90 \mathrm{mg} / \mathrm{kg} /$ day in the first year. The retention rate for US patients at 2 years was nearly $80 \%$ [11]. Similar results were found in a singlecenter study of clobazam with children from Seoul, Korea, with LGS, which reported retention rates of $80.2 \%$ at 2 years and $76.6 \%$ at 3 years [21].

The switch from clonazepam to clobazam should be considered in light of relevant pharmacokinetic and pharmacodynamic factors of these compounds. One of the important determinants of brain uptake is relative lipophilicity, often estimated by the octanol-to-water partition ratios. Via this in vitro measure, clobazam is much less lipophilic than diazepam (ratio of 9 for clobazam versus 309 for diazepam). Similar data for clonazepam are not readily available $[22,23]$. However, the retention times for diazepam, clonazepam, and clobazam in a reverse-phase, high-performance liquid chromatography (HPLC) system using a neutral mobile phase are 31.4 minutes, 14.9 minutes, and 13.1 minutes, suggesting that clonazepam and clobazam are fairly similar in this measure of lipophilicity. The retention time for $\mathrm{N}$ desmethylclobazam is 12 , again not far from the times for clonazepam or clobazam. Of the in vitro estimates, available data [22,23] suggest that HPLC retention times correlated better with in vivo unbound volumes of distribution for a series of benzodiazepines. Arendt and colleagues [22] showed that despite the difference in lipophilicity, the cerebrospinal fluid-to-plasma ratio for clobazam was greater than that for diazepam, a result they attributed to the fact that clobazam is less protein-bound than diazepam ( $83 \%$ for clobazam versus 99\% for diazepam), since it is the unbound fraction that transits the blood-brain barrier. Unfortunately, data were not provided for clonazepam in this report. The protein binding of clonazepam is reported to be $85 \%$ in a different article [24], and these values are also in agreement with those reported by Riss et al. [25]. Thus, based on a combination of lipophilicity and protein binding, the central nervous system penetration of clonazepam and clobazam should be comparable.

The binding affinities $\left(K_{i}\right.$ in $\left.n M\right)$ for clonazepam, clobazam, and $N$-desmethylclobazam to rat brain homogenates have been determined to be $0.26,151$, and 153 [10], respectively, corresponding to the greater potency of clonazepam over clobazam on a mg basis as used in patients. In the same report, Jensen et al. also showed that the $\mathrm{pK}_{\mathrm{i}}$ values for clobazam and $N$ - desmethylclobazam for $\alpha_{2}$-subunit-containing receptors exceeded that for those containing $\alpha_{1}$ subunits, whereas these values were similar for clonazepam. Zolpidem, however, exhibited the greatest $\mathrm{pK}_{\mathrm{i}}$ values for receptors containing $\alpha_{1}$ subunits. These data would explain, at least in part, why clobazam may be much less sedating than clonazepam, as observed by Nicholson [16] nearly 35 years ago.

For drug disposition, clobazam (estimated half-life of 36 hours) undergoes hepatic metabolism to $N$-desmethylclobazam (estimated half-life of 80 hours). Because of the lower clearance, the latter achieves an approximately 10to 15 -fold steady concentration by approximately 4 weeks after initiation of therapy [26]. Both clobazam and $\mathrm{N}$-desmethylclobazam show a bimodal response to chloride conductance when applied to cultured cerebral neurons in the presence of $10-\mu \mathrm{M}$ GABA, peaking around $3 \mu \mathrm{M}$ clobazam or $N$-desmethylclobazam, and declining substantially at $10 \mu \mathrm{M}$ concentrations of either allosteric agonist [27]. Thus, under clinical conditions, $N$ - desmethylclobazam can appear less potent. The important finding, however, is that the concentration of $\mathrm{N}$-desmethylclobazam stabilizes 4 weeks after initiation of therapy. A study of drug-drug interactions found no clinically meaningful drug-drug interactions between clobazam and drugs metabolized by CYP3A4, CYP2C19, CYP1A2, or CYP2C9 [28]. The authors concluded that clobazam may be administered safely as adjunctive therapy in patients with LGS, without meaningful changes in clobazam pharmacokinetics that would require dosage adjustments.

\section{Conclusions}

The literature reviewed thus supports the transition strategies highlighted in the cases described. A rough estimate of the final dosage of clobazam for each $\mathrm{mg}$ of clonazepam under substitution is likely to be at least 10fold, probably closer to 15 -fold for many patients, and as high as 20-fold for a few. While there is prudent caution in delaying the weaning of clonazepam until significant clobazam is already onboard from the point of view of breakthrough seizures, patients - as well as their families and caregivers - should be counseled to expect increased sedation transiently with that approach. Given the common clinical experience with the severity of withdrawal seizures associated with removal of clonazepam, it would be wise not to begin aggressive removal of clonazepam from the patients' regimens as soon as clobazam is initiated. These guidelines are general, because if the patient is also receiving other medications acting via GABA mechanisms, the removal of clonazepam is likely to be tolerated slightly better and breakthrough seizures are less likely or may be milder should such 
seizures intervene. A transition from clonazepam to clobazam, in consideration of patients' individual seizure patterns and vulnerabilities, with careful application of the principles described in this article, and with close clinical observation, can be reasonably expected to improve seizure control and enhance quality of life for patients and their families.

\section{Consent}

Written informed consent was obtained from both of the adult patients and the parents of the child for publication in this case series. Copies of the written consents are available for review by the Editor-in-Chief of this journal.

\section{Abbreviations}

CYP1A2: Cytochrome P450 isozyme 1A2; CYP2C9: Cytochrome P450 isozyme 2C9; CYP2C19: Cytochrome P450 isozyme 2C19; CYP3A4: Cytochrome P450 isozyme 3A4; EEG: Electroencephalograph; FDA: Food and drug administration; GABA: $\gamma$-aminobutyric acid; GTC: Generalized tonic-clonic; HPLC: High-performance liquid chromatography; $K_{\text {: }}$ Inhibition constant; LGS: Lennox-Gastaut syndrome; MRI: Magnetic resonance imaging; PI: Protective index; VNS: Vagal nerve stimulation.

\section{Competing interests}

RS serves on scientific advisory boards for and has received honoraria and funding for travel from UCB Pharma, Sunovion, Upsher-Smith Laboratories, Supernus, Lundbeck LLC, and Acorda Therapeutics; and serves on speakers' bureaus for and has received speaker honoraria from UCB, GlaxoSmithKline, Cyberonics, Supernus and Lundbeck. He receives funding from NIH NINDS NS065783 (coinvestigator), NS045911 (site PI CAE Study), P20 NS080181 (Epilepsy Research Centers without Walls), as well as from Pfizer (Lyrica pediatric partial seizures trial) and Bluebird Bio trial for gene therapy for ALD. He has received royalties for books from CRC Press and Demos Publishers. $\mathrm{SC}$ received funding for consulting, serving on scientific advisory boards or speakers' bureaus, or other activities from UCB Pharma, Supernus, Eisai, Lundbeck LLC, Upsher-Smith Laboratories, SK Life Science, and Acorda. MSP has served on speakers' bureaus for Lundbeck LLC, Quest Diagnostics, and Transgenomics, as well as advisory boards for Lundbeck LLC and Eisai. RK has served on the speakers' bureaus for Lundbeck LLC and UCB Pharma. SS has served on advisory boards for Lundbeck LLC, Acorda, and Upsher-Smith Laboratories and on a speaker's bureau for Cyberonics. He has received research grants from Cyberonics, Upsher-Smith Laboratories, and UCB Pharm, and is a board member for the American Board of Registration of Electroencephalographic and Evoked Potential Technologists and the American Board for Clinical Neurophysiology.

\section{Authors' contributions}

These cases, and others, were prepared, reviewed, and discussed by the authors at a Case-Report Colloquium July 27, 2013, in Chicago, USA. The meeting was funded by Lundbeck LLC (Deerfield, IL, USA), and the authors received honoraria for preparing their research and attending the meeting. The authors received no compensation to prepare this article. RS chaired the meeting and drafted the Introduction and Discussion sections. MSP provided and drafted Case 1, and SC provided and drafted Cases 2 and 3. The first draft of this article was prepared by RS, SC, and MSP. All authors reviewed the article for important intellectual content and confirm that they meet the current International Committee of Medical Journal Editors' four requirements for authorship. All authors read and approved the final manuscript.

\section{Acknowledgments}

Writing assistance and editorial support during manuscript preparation and revision were provided by Michael A. Nissen, ELS, of Lundbeck LLC (Deerfield, IL, USA). Editorial support and article processing fees were funded by Lundbeck LLC.

\section{Author details}

'Division of Pediatric Neurology, David Geffen School of Medicine at UCLA, University of California-Los Angeles, Room 22-474 MDCC, Los Angeles, CA 90095-1752, USA. ${ }^{2}$ Barrow Neurological Institute, 500 W. Thomas Road, Phoenix, AZ 85013, USA. ${ }^{3}$ Jane and John Justin Neuroscience Center, Cook Children's Medical Center, 4th Floor, 1500 Cooper Street, Fort Worth, TX 76104, USA. ${ }^{4}$ Department of Neurology, NYU Epilepsy Center, New York University School of Medicine, 223 East 34 Street, New York, NY 10016, USA. ${ }^{5}$ Neurology Residency Program, Epilepsy Monitoring Unit, Duke University Medical Center, DUMC 102350, Durham, NC 27710, USA.

Received: 18 August 2014 Accepted: 15 October 2014

Published: 16 December 2014

\section{References}

1. Gastaut H, Roger J, Soulayrol R, Lob H, Tassinari CA: L'action du diazepam (Valium) dans le traitement des formes non-convulsives de l'épilepsie généralisée. Rev Neurol (Paris) 1965, 112:99-118.

2. Gastaut $H$, Naquet R, Poiré $R$, Tassinari CA: Treatment of status epilepticus with diazepam (Valium). Epilepsia 1965, 6:167-182.

3. Gastaut H, Courjon J, Poire R, Weber M: Treatment of status epilepticus with a new benzodiazepine more active than diazepam. Epilepsia 1971, 12:197-214.

4. Brown TR: Clonazepam: a review of a new anticonvulsant drug. Arch Neurol 1976, 33:326-332.

5. Klonopin (Clonazepam), [prescribing information]. South San Francisco, CA, USA: Genentech; 2010.

6. Ng YT, Conry JA, Drummond R, Stolle J, Weinberg MA, OV-1012 Study Investigators: Randomized, phase III study results of clobazam in Lennox-Gastaut syndrome. Neurology 2011, 77:1473-1481.

7. Sankar R, Holmes GL: Mechanisms of action for the commonly used antiepileptic drugs: relevance to antiepileptic drug-associated neurobehavioral adverse effects. J Child Neurol 2004, 19(Suppl 1):S6-S14.

8. Glauser TA: Effects of antiepileptic medications on psychiatric and behavioral comorbidities in children and adolescents with epilepsy. Epilepsy Behav 2004, 5(Suppl 3):S25-S32.

9. Sankar R: $G A B A_{A}$ receptor physiology and its relationship to the mechanism of action of the 1,5-benzodiazepine clobazam. CNS Drugs 2012, 26:229-244.

10. Jensen HS, Nichol K, Lee D, Ebert B: Clobazam and its active metabolite $\mathrm{N}$ - desmethylclobazam display significantly greater affinities for a2- versus a1-GABA $A_{A}$-receptor complexes. PLoS One 2014, 9:e88456.

11. Conry JA, Ng YT, Kernitsky L, Mitchell WG, Veidemanis R, Drummond R, Isojarvi J, Lee D, Paolicchi JM, and on behalf of the OV-1004 Study Investigators: Stable dosages of clobazam for Lennox-Gastaut syndrome are associated with sustained drop-seizure and total-seizure improvements over 3 years. Epilepsia 2014, 55:558-567.

12. Shenoy AK, Miyahara JT, Swinyard EA, Kupferberg HJ: Comparative anticonvulsant activity and neurotoxicity of clobazam, diazepam, phenobarbital, and valproate in mice and rats. Epilepsia 1982, 23:399-408.

13. Stéru $L$, Chermat R, Millet B, Mico JA, Simon P: Comparative study in mice of ten 1,4-benzodiazepines and of clobazam: anticonvulsant, anxiolytic, sedative, and myorelaxant effects. Epilepsia 1986, 27(Suppl 1):S14-S17.

14. Tietz El, Rosenberg HC, Chiu TH: A comparison of the anticonvulsant effects of 1,4- and 1,5-benzodiazepines in the amygdala-kindled rat and their effects on motor function. Epilepsy Res 1989, 3:31-40.

15. Borland RG, Nicholson AN: Immediate effects on human performance of a 1,5- benzodiazepine (clobazam) compared with the 1,4-benzodiazepines, chlordiazepoxide hydrochloride and diazepam. Br J Clin Pharmacol 1975, 2:215-221.

16. Nicholson AN: Differential effects of the 1,4- and 1,5-benzodiazepines on performance in healthy man. Br J Clin Pharmacol 1979, 7(Suppl 1):S83-S84.

17. Rosenberg HC, Tietz El, Chiu TH: Tolerance to anticonvulsant effects of diazepam, clonazepam, and clobazam in amygdala-kindled rats. Epilepsia 1989, 30:276-285.

18. Löscher W, Rundfeldt C, Hönack D, Ebert U: Long-term studies on anticonvulsant tolerance and withdrawal characteristics of benzodiazepine receptor ligands in different seizure models in mice. I. Comparison of diazepam, clonazepam, clobazam and abecarnil. J Pharmacol Exp Ther 1996, 279:561-572. 
19. Munn R, Farrell K: Open study of clobazam in refractory epilepsy. Pediatr Neurol 1993, 9:465-469.

20. Singh A, Guberman AH, Boisvert D: Clobazam in long-term epilepsy treatment: sustained responders versus those developing tolerance. Epilepsia 1995, 36:798-803.

21. Lee EH, Yum MS, Choi HW, Ko TS: Long-term use of clobazam in Lennox-Gastaut syndrome: experience in a single tertiary epilepsy center. Clin Neuropharmacol 2013, 36:4-7.

22. Arendt RM, Greenblatt DJ, de Jong RH, Bonin JD, Abernethy DR, Ehrenberg BL, Giles HG, Sellers EM, Shader RI: In vitro correlates of benzodiazepine cerebrospinal fluid uptake, pharmacodynamic action and peripheral distribution. J Pharmacol Exp Ther 1983, 227:98-106.

23. Greenblatt DJ, Arendt RM, Abernethy DR, Giles HG, Sellers EM, Shader Rl: In vitro quantitation of benzodiazepine lipophilicity: relation to in vivo distribution. Br J Anaesth 1983, 55:985-989.

24. Pacifici GM, Viani A, Rizzo G, Carrai M, Rane A: Plasma protein binding of clonazepam in hepatic and renal insufficiency and after hemodialysis. Ther Drug Monit 1987, 9:369-373.

25. Riss J, Cloyd J, Gates J, Collins S: Benzodiazepines in epilepsy: pharmacology and pharmacokinetics. Acta Neurol Scand 2008, 118:69-86.

26. Rupp W, Badian M, Christ O, Hajdu P, Kulkarni RD, Taeuber K, Uihlein M, Bender $\mathrm{R}$, Vanderbeke O: Pharmacokinetics of single and multiple doses of clobazam in humans. Br J Clin Pharmacol 1979, 7(Suppl 1):S51-S57.

27. Nakamura F, Suzuki S, Nishimura S, Yagi K, Seino M: Effects of clobazam and its active metabolite on GABA-activated currents in rat cerebral neurons in culture. Epilepsia 1996, 37:728-735.

28. Walzer M, Bekersky I, Blum RA, Tolbert D: Pharmacokinetic drug interactions between clobazam and drugs metabolized by cytochrome P450 isoenzymes. Pharmacotherapy 2012, 32:340-353.

doi:10.1186/1752-1947-8-429

Cite this article as: Sankar et al.: Clinical considerations in transitioning patients with epilepsy from clonazepam to clobazam: a case series. Journal of Medical Case Reports 2014 8:429.

\section{Submit your next manuscript to BioMed Central and take full advantage of:}

- Convenient online submission

- Thorough peer review

- No space constraints or color figure charges

- Immediate publication on acceptance

- Inclusion in PubMed, CAS, Scopus and Google Scholar

- Research which is freely available for redistribution 